\section{Mortality after colectomy (elective or emergency) or no colectomy for patients with IBD}

Total or partial colectomy is often necessary at some stage for patients with IBD. Small, short-term studies have reported that mortality after elective colectomy is low, but large, longer-term studies are lacking. Roberts et al., therefore, conducted a large study to compare 3-year mortality after elective colectomy, emergency colectomy or no colectomy in patients hospitalized for IBD.

The study analyzed record-linked inpatient and mortality data for 23,464 patients from the Oxford region (1968-1999) and England (19982003) who had been hospitalized for $>3$ days for IBD. In total, 5,480 patients underwent colectomy. Three-year mortality was increased after no colectomy or emergency colectomy compared with that after elective colectomy; this finding was nonsignificant for the Oxford data, but significant even after adjustment for inpatient comorbidity for the larger England data. Elective colectomy carried an increased risk of mortality relative to the general population, but only for the first few months. Of concern, 3 year risk of mortality was almost as high for patients who did not undergo colectomy as for those who underwent emergency colectomy.

It remains to be seen whether new immunosuppressive drugs reduce the colectomy rate and mortality in patients presenting acutely with severe colitis. For those with chronic disease the findings indicate that in appropriate cases it is preferable to undertake elective colectomy earlier rather than risk emergency colectomy, which carries a much poorer prognosis. The authors suggest that it may be beneficial to lower the threshold for elective colectomy for patients with IBD.

Original article Roberts SE et al. (2007) Mortality in patients with and without colectomy admitted to hospital for ulcerative colitis and Crohn's disease: record linkage studies. BMJ 335: 1033

\section{Cetuximab improves survival in patients with colorectal cancer}

Cetuximab is a monoclonal antibody with activity against colorectal cancers that overexpress epidermal growth factor receptor. This agent blocks epidermal growth factor receptor signaling and inhibits tumor growth.
Jonker et al. conducted a randomized trial from December 2003 to August 2005 to investigate the effects of cetuximab in patients with advanced colorectal cancer. Patients enrolled in the trial $(n=572)$ were unresponsive or had contraindications to irinotecan, oxaliplatin and fluoropyrimidine therapy. All received best supportive care; 287 were also treated with cetuximab.

The median duration of cetuximab treatment was 8.1 weeks and of follow-up was 14.6 months. Cetuximab therapy was associated with a significant improvement in overall survival and in progression-free survival, compared with best supportive care alone. The median overall survival was 6.1 months in the cetuximab group and 4.6 months in the group receiving only supportive care. Rash was seen in 255 of the patients treated with cetuximab. Increasing grade of rash severity was associated with improved median overall survival: 2.6 months with no rash, 4.8 months with grade 1 rash, and 8.4 months with grade 2 or higher rash.

The authors conclude that cetuximab can help patients with advanced colorectal cancer for whom other treatments have been unsuccessful. The authors suggest that the occurrence of rash could be used as a predictive biomarker to identify patients most likely to benefit from this treatment, but validation of this role is needed.

Original article Jonker DJ et al. (2007) Cetuximab for the treatment of colorectal cancer. N Engl J Med 357: 2040-2048

\section{Malignant and benign IPMNs can be differentiated accurately by the 18F-FDG PET technique}

Intraductal papillary mucinous neoplasms (IPMNs) of the pancreas frequently occur in people aged $>70$ years. Differentiation of benign and malignant IPMNs is important when making surgical and treatment decisions concerning such elderly patients, but CT and MRI do not provide reliable information.

Sperti et al. previously reported that PET with fluorine-18-labeled fluorodeoxyglucose $\left({ }^{18} \mathrm{~F}-\mathrm{FDG}\right.$ PET), a technique that visualizes the rate of glucose uptake by tumor cells, could discriminate accurately between malignant and benign cystic tumors of the pancreas. 